\title{
A Non-Archimedean Approach to Prolongation Theory
}

\author{
H. N. VAN ECK \\ Twente University of Technology, Department of Applied Mathematics, P.O. Box 217. \\ 7500 AE Enschede. The Netherlands
}

(Received: 25 June 1986)

\begin{abstract}
Some evolution equations possess infinite-dimensional prolongation Lie algebras which can be made finite-dimensional by using a bigger (non-Archimedean) field. The advantage of this is that convergence problems hardly exist in such a field. Besides that, the accompanying Lie groups can be easily constructed.
\end{abstract}

The prolongation theory of Wahlquist and Estabrook [1] can be described as follows: Find a 1-form $\omega=A \mathrm{~d} x+B \mathrm{~d} t$, defined on the $(x, t)$-space with values in a Lie algebra g such that

$$
\mathrm{d} \omega+\frac{1}{2} \omega \wedge \omega=\left(-\frac{\partial A}{\partial t}+\frac{\partial B}{\partial x}+[A, B]\right) \mathrm{d} x \wedge \mathrm{d} t
$$

is a linear combination of the 2-forms which describe the evolution equation in question. If $u$ is an analytic solution of this equation, then $A$ and $B$ are analytic functions of $u$ and its $x$-derivatives. $\mathbf{g}$ is called a prolongation algebra and is found, as a presentation, as a part of the prolongation problem.

In this formulation the prolongation variables seem to have disappeared but they turn out to be coordinates of a Lie group $G$ which has (a completion of) $g$ as a Lie algebra.

One can find conservation laws by using the following theorem.

THEOREM (th. 5 of 4.6 of [3]). Let $G$ be a Lie group (local or global), $M$ an analytic manifold, $\omega$ a 1-form on $M$ with values in the Lie algebra $\mathrm{g}$ of $G$ such that $\mathrm{d} \omega+\frac{1}{2} \omega \wedge \omega=0$, then for every $x \in M$ and $g \in G$ there is an analytic map $f$, defined on an open neighbourhood of $x$, with values in $G$, such that $f(x)=g$ and that $f^{-1} \mathrm{~d} f=\omega$. Two such $f^{\prime}$ 's coincide in the neighbourhood of $x$.

REMARK. $G$ and $M$ are analytic manifolds over the same field $K$ where $K$ can be $\mathbb{R}$, $\mathbb{C}$, or a complete non-Archimedean field. An example of the latter will be given below.

We shall now construct $f$ explicitly for the Korteweg-de Vries equation

$$
u_{t}+12 u u_{x}+u_{x x x}=0
$$

the nonlinear Schrödinger equation

$$
i \psi_{t}+\psi_{x x}-\frac{1}{2} \bar{\psi} \psi^{2}=0
$$


and the Burgers equation

$$
q_{t}+2 q q_{x}+q_{x x}=0 .
$$

Here the following fact is used: if $b_{1}, \ldots, b_{n}$ is a basis of the Lie algebra $\mathbf{g}$, then $\mathrm{e}^{\alpha_{1} b_{1}} \ldots \mathrm{e}^{\alpha_{n} b_{n}}$ sweeps out from a neighbourhood of 1 in $G$ when the $x_{i}$ progress through a neighbourhood of 0 in $K$ [3]. Moreover, the formula for $U$ and $V \in \mathbf{g}$

$$
\mathrm{e}^{U} V \mathrm{e}^{-U}=\mathrm{e}^{\mathrm{ad} U}(V)=V+[U, V]+\frac{1}{2 !}[U,[U, V]]+\cdots
$$

is requisite [2].

$K d V$ : The solution of the prolongation problem is

$$
A=-2 X_{1}-2 u X_{2}-3 u^{2} X_{3}
$$

and

$$
B=2\left(u_{x x}+6 u^{2}\right) X_{2}+3\left(8 u^{3}-u_{x}^{2}+2 u u_{x x}\right) X_{3}-8 X_{4}-8 u X_{5}-4 u^{2} X_{6}-4 u_{x} X_{7}
$$

(see [1]) and the prolongation algebra $\mathbf{g}=H \times\left(A_{1} \otimes \mathbb{C}[T]\right) . H$ has a basis $\left(r_{-3}, r_{-1}, r_{0}, r_{1}, r_{3}\right)$ with multiplication table $\left[r_{1}, r_{-1}\right]=\left[r_{-3}, r_{3}\right]=-r_{0}$; the other commutators being zero.

$A_{1}$ has a basis $(h, y, z)$ with commutatorable $[h, y]=2 y,[h, z]=-2 z$, and $[y, z]=h$. $\mathbb{C}[T]$ is the ring of polynomials in the indeterminate $T$ with complex coefficients. Every element of $H$ commutes with any of $A_{1} \otimes \mathbb{C}[T]$. Details are found in [4] and [5].

We can decompose $X_{1}, \ldots, X_{7}$ :

$$
\begin{aligned}
& X_{1}=r_{1}-\frac{1}{2} y+\frac{1}{2} T z, \quad X_{2}=r_{-1}+z, \quad X_{3}=r_{-3}, \quad X_{4}=r_{3}-\frac{1}{2} T y+\frac{1}{2} T^{2} z, \\
& X_{5}=\frac{1}{2} y+\frac{1}{2} T z, \quad X_{6}=z, \quad X_{7}=r_{0}+\frac{1}{2} h .
\end{aligned}
$$

We can split $A$ and $B$ accordingly, so we can look upon the $\mathrm{KdV}$ as having two prolongation algebras, $H$ and $A_{1} \otimes \mathbb{C}[T]$.

$K d V$ with $\mathbf{g}=H$ :

$$
\begin{aligned}
& A=-2 r_{1}-2 u r_{-1}-3 u^{2} r_{-3}, \\
& B=2\left(u_{x x}+6 u^{2}\right) r_{-1}+3\left(8 u^{3}-u_{x}^{2}+2 u u_{x x}\right) r_{-3}-8 r_{3}-4 u_{x} r_{0} .
\end{aligned}
$$

We can easily check that

$$
-\frac{\partial A}{\partial t}+\frac{\partial B}{\partial x}+[A, B]=0
$$

if and only if $u$ satisfies the KdV. Put

$$
f(x, t)=\mathrm{e}^{\alpha_{3} r_{-3}} \mathrm{e}^{\alpha_{1} r_{-1}} \mathrm{e}^{\beta_{0} r_{0}} \mathrm{e}^{\gamma_{1} r_{1}} \mathrm{e}^{\gamma / r_{3}}
$$


then $f^{-1} \mathrm{~d} f=\omega$ is translated into

$$
f^{-1} \frac{\partial f}{\partial x}=A \text { and } f^{-1} \frac{\partial f}{\partial t}=B
$$

We find

$$
\alpha_{3 x}\left(r_{-3}-\gamma_{3} r_{0}\right)+\alpha_{1 x}\left(r_{\ldots 1}+\gamma_{1} r_{0}\right)+\beta_{0 x} r_{0}+\gamma_{1 x} r_{1}+\gamma_{3 x} r_{3}=A
$$

and the same equation with $x$ replaced by $t$ and $A$ by $B$. Sorting out yields

$$
\begin{aligned}
& x_{3 x}=-3 u^{2}, \quad \alpha_{3 t}=-3 u_{x}^{2}+24 u^{3}+6 u u_{x x} ; \quad \alpha_{1 x}=-2 u, \quad \alpha_{1 t}=12 u^{2}+2 u_{x x} ; \\
& \gamma_{1 x}=-2, \quad \gamma_{1 t}=0, \quad \text { so } \gamma_{1}=-2 x ; \quad \gamma_{3 x}=0, \quad \gamma_{3 t}=-8, \quad \text { so } \gamma_{3}=-8 t ; \\
& \beta_{0 x}=-8 t \alpha_{3 x}+2 x \alpha_{1 x}, \quad \beta_{0 t}=-4 u_{x}-8 t \alpha_{3 t}+2 x \alpha_{1 t} .
\end{aligned}
$$

We get the following conservation laws

$$
\int u^{2} \mathrm{~d} x \text { from } \alpha_{3}, \quad \int u \mathrm{~d} x \text { from } \alpha_{1} \text { and } \int\left(6 t u^{2}-x u\right) \text { from } \beta_{0} .
$$

The last one is the same one as described in Section 6 of [6].

$K d V$ with $\mathbf{g}=A_{1} \otimes \mathbb{C}[T]:$

$$
A=y-(2 u+T) z \text { and } B=\left(2 u_{x x}+8 u^{2}-4 T^{2}-4 u T\right) z+(4 T-4 u) y-2 u_{x} h .
$$

Again

$$
-\frac{\partial A}{\partial t}+\frac{\partial B}{\partial x}+[A, B]=0
$$

if and only if $u$ satisfies the $\mathrm{KdV}$. Now $\mathbf{g}$ is an infinite-dimensional algebra over $\mathbb{C}$. We make it finite-dimensional, not by chopping an infinite tail off it but by enlarging it. In fact, we enlarge the ring $\mathbb{C}[T]$ to the field $\mathbb{C}((T))$, where the elements are formal Laurent series in $T$, with coefficients in $\mathbb{C}$, having a finite principal part.

If $s=\sum_{n=m}^{\infty} a_{n} T^{n}$ with $a_{m} \neq 0$ then $\|s\|=\mathrm{e}^{-m} \cdot\|\|$ is a norm on $\mathbb{C}((T))$ of which the triangle inequality is sharpened to

$$
\left\|s_{1}+s_{2}\right\| \leq \sup \left(|| s_{1}\|,\| s_{2} \|\right) \text {. }
$$

Sometimes such a norm is called an ultranorm. In this norm, $\mathbb{C}((T))$ is complete and is the promised example of a non-Archimedean complete field. $\mathbf{g}$ has thus become a three-dimensional algebra over $\mathbb{C}((T))$ - an $\operatorname{sl}(2)$. In $\mathbb{C}((T))$ there exist the notions of 'differentiation of a function', 'analytic functions' and 'manifolds' [11], and 'Lie groups' [3].

Let $f(x, t)=\mathrm{e}^{\alpha h} \mathrm{e}^{\beta y} \mathrm{e}^{y z}$ then $f^{-1} \mathrm{~d} f=\omega$ becomes

$2 x_{x} \beta+\beta_{x}=1, \quad \alpha_{x}(1+2 \beta \gamma)+\beta_{x} \gamma=0, \quad-2 \alpha_{x}\left(\gamma+\beta \gamma^{2}\right)-\beta_{x} \gamma^{2}+\gamma_{x}=-T-2 u$,

$2 \alpha_{t} \beta+\beta_{t}=4 T-4 u, \quad \alpha_{t}(1+2 \beta \gamma)+\beta_{t} \gamma=-2 u_{x} \quad$ and

$-2 \alpha_{t}\left(\gamma+\beta \gamma^{2}\right)-\beta_{t} \gamma^{2}+\gamma_{t}=2 u_{x x}+8 u^{2}-4 T^{2}-4 u T$. 
From the first and fourth equations, it follows that

$$
\left(\beta \mathrm{e}^{2 \alpha}\right)_{x}=\mathrm{e}^{2 \alpha} \text { and }\left(\beta \mathrm{e}^{2 \alpha}\right)_{t}=(4 T-4 u) \mathrm{e}^{2 \alpha},
$$

so $\mathrm{e}^{2 \alpha}$ can be viewed as a conservation density. We return to it below.

If we insert the first equation of (1) into the second, resp. the third, we get

$$
\alpha_{x}=-\gamma \text { and } \gamma_{x}+\gamma^{2}=-T-2 u .
$$

From the $t$-equations of (1) we can derive

$$
\begin{aligned}
& \alpha_{t}+(4 T-4 u) \gamma=-2 u_{x}, \\
& \gamma_{t}+4 u_{x} \gamma+(4 T-4 u) \gamma^{2}=2 u_{x x}+8 u^{2}-4 T^{2}-4 u T .
\end{aligned}
$$

If we eliminate $u$ from (3) and (4) we get

$$
\gamma_{t}+\gamma_{x x x}-6 \gamma^{2} \gamma_{x}-6 T \gamma_{x}=0
$$

the so-called modified $K d V$ equation.

We now give a few consequences of this equation.

1. Note that $-\gamma$ satisfies (5) too. If $u^{\prime}$ is such that $-\gamma_{x}+\gamma^{2}=-T-2 u^{\prime}$ (see second equation of (3)), then $-\gamma$ and $u^{\prime}$ satisfy the second equation of (4). That means that we can find an $f^{\prime}$ such that $f^{\prime-1} \mathrm{~d} f^{\prime}=\omega^{\prime}$, where $\omega^{\prime}$ is made from $\omega$ by replacing $u$ with $u^{\prime}$. It follows that $u^{\prime}$ is a solution of the $\mathrm{KdV}$. The idea of using $-\gamma$ in order to find a new solution of the $\mathrm{KdV}$ was brought in by Martini (Twente University of Technology).

2. Replace $T$ by $-\left(1 / 4 T^{\prime 2}\right)$ and to things in $\mathbb{C}\left(\left(T^{\prime}\right)\right)$. The equation $\gamma_{x}+\gamma^{2}=1 / 4 T^{\prime 2}$ has $\gamma=1 / 2 T^{\prime}$ as a solution. Now put $\gamma=\left(1 / 2 T^{\prime}\right)+T^{\prime} \gamma^{\prime}$, then the second equation of (3) becomes

$$
T^{\prime} \gamma_{x}^{\prime}+\gamma^{\prime}+T^{\prime 2} \gamma^{\prime 2}=-2 u .
$$

If $u$ is a real analytic solution of the $\mathrm{KdV}$, then it is analytic in the neighbourhood $\|x\|<1$, $|t|<1$ of $\mathbb{C}\left(\left(T^{\prime}\right)\right)^{2}$. Formula (6) has an analytic solution in the same neighbourhood. Miura $[6,7]$ found this as follows. Assume that for $x$ and $t \in \mathbb{R}$, $\gamma^{\prime}=\Sigma_{n=0}^{\infty} \gamma_{n} T^{\prime n}$ with $\gamma_{n}(x, t) \in \mathbb{R}$. We can solve $\gamma_{0}, \gamma_{1}, \gamma_{2}, \ldots$ consecutively from (6). They are polynomials in $u$ and its derivatives. Miura proved that the $\gamma^{\prime}$ thus found satisfies the equation derived from (5) by substituting $\left(1 / 2 T^{\prime}\right)+T^{\prime} \gamma^{\prime}$ for $\gamma$. Because $\alpha_{x}=-\gamma$ (see (3)), all $\gamma_{n}$ are conservation densities, so that $\int_{x \in \mathbb{R}} \gamma^{\prime}(x, t) \mathrm{d} x$, with the obvious meaning, is time independent. It is clear that $\gamma^{\prime}$ is analytic in the abovementioned neighbourhood.

3. Equation (3) can be combined to $-\alpha_{x x}+\alpha_{x}^{2}=-T-2 u$ and multiplication by $\mathrm{e}^{-\alpha}$ gives, with $\partial:=\partial / \partial x$,

$$
\left(\partial^{2}+2 u\right) \mathrm{e}^{-x}=-T \mathrm{e}^{\cdots \alpha} .
$$

If we combine the first equations of (3) and (4) and multiply with $e^{-\alpha}$, we get

$$
\left(-\mathrm{e}^{-\alpha}\right)_{t}+(4 T-4 u) \partial \mathrm{e}^{-\alpha}+2 u_{x} \mathrm{e}^{-\alpha}=0 .
$$


With the help of (7), we can eliminate $T$ from (8) and get

$$
\left(\mathrm{e}^{-x}\right)_{t}=\left(-4 \partial^{3}-6 u_{x}-12 u \partial\right) \mathrm{e}^{-\alpha} .
$$

Set

$$
\phi=\mathrm{e}^{-\alpha}, \quad L=\partial^{2}+2 u \text { and } P=-4 \partial^{3}-6 u_{x}-12 u \partial
$$

then (7) and (9) read as $L \phi=-T \phi$ and $\phi_{t}=P \phi$. Differentiation gives

$$
L_{t} \phi+L \phi_{t}=-T \phi_{t} \quad \text { or } \quad L_{t} \phi+L P \phi=-T P \phi=P L \phi
$$

so that $L_{t} \phi=[P, L] \phi$. Indeed, $L_{t}=[P, L]$ by direct computation.

From [8] it follows that the spectral data of $L$ are time invariant in the KdV flow.

We remarked before that $\mathrm{e}^{2 x}$ is a conservation density. Now $\mathrm{e}^{2 a}=\phi^{-2}$ so there is a link with the theorem on page 92 of [9].

4. Equation (7) has a close relationship with the Lenard sequence [10]. Applying Lemma 3.8 of this reference to (7), one gets

$$
\left(\partial^{3}+8 u \partial+4 u_{x}\right) \phi^{2}=T^{\prime-2} \hat{\partial} \phi^{2} .
$$

If for $x, t \in \mathbb{R} ; \phi^{2}=\Sigma_{n \in \mathbb{Z}} \phi_{n} T^{\prime n}$ with $\phi_{n}(x, t) \in \mathbb{R}$ then

$$
\left(\partial^{3}+8 u \partial+4 u_{x}\right) \phi_{n}=\partial \phi_{n+2} .
$$

5. The prolongation variables of [1] are coordinates of the group $G$. In the notation of [1] we have

$$
\alpha_{3}=y_{6}, \quad \alpha_{1}=y_{7}, \quad \alpha=y_{3}, \quad \gamma=y_{8} \quad \text { and } \beta \mathrm{e}^{2 \alpha}=y_{2} .
$$

A final remark about $\gamma$ : if we factorize the operator $\partial^{2}+2 u+T$ (see [7]), we get $\partial^{2}+2 u+T=(\partial+\gamma)(\partial-\gamma)$, which gives another point of view of the Băcklund transformation described in consequence 1 above.

The important role of $\gamma$ (and of $\alpha$ ) in the prolongation of the $\mathrm{KdV}$ has now become clear. We shall now say something about the role of $\beta$. From the first equations of (1) and (3), it follows that $\beta_{x}-2 \gamma \beta=1$.

Substitution of $\gamma^{\prime} T^{\prime}+\left(1 / 2 T^{\prime}\right)$ for $\gamma$ (see consequence 2$)$ yields

$$
\left(\partial-2 \gamma^{\prime} T^{\prime}-\frac{1}{T^{\prime}}\right) \beta=1 \quad \text { or } \quad \beta=-\sum_{n=0}^{\infty} T^{\prime n+1}\left(\partial-2 T^{\prime} \gamma^{\prime}\right)^{n}(1) \text {. }
$$

From this it follows that

$$
\beta=-T^{\prime}-4 u T^{\prime 3}-\left(24 u^{2}+4 u_{x x}\right) T^{\prime 5}-\cdots .
$$

We can solve the equation $\beta_{x}-2 \gamma \beta=1$ for $\gamma$. If we put the result in the second equation of (3) we get

$$
-2 \beta \beta_{x x}+\beta_{x}^{2}-4 \beta^{2}(2 u+T)=1 .
$$

If we compare this with Equation (2.6) of [16] we find that $\beta$ is equal to $R(x ; \xi)$, the restriction to the diagonal $x=y$ of the resolvent of the Schrodinger operator. 
Finally, $\beta$ can be used to build a hierarchy of generalized $\mathrm{KdV}$ equations. The relation $-A_{t}+B_{x}+[A, B]=0$ can be looked upon as an equation for $B$ by given $A$. If $A$ remains equal to $y-(2 u+T) z$ and if $B=b_{1} h+b_{2} y+b_{3} z$, we find that

$$
\partial b_{1}+b_{3}+(2 u+T) b_{2}=0, \quad \partial b_{2}-2 b_{1}=0
$$

and

$$
u_{t}=\frac{1}{4}\left(\hat{\partial}^{3}+8 u \hat{\partial}-T^{\prime-2} \partial+4 u_{x}\right) b_{2} .
$$

The right-hand member of this equation can be made homogeneous in $T^{\prime}$ as follows. By differentiation of the second-order equation for $\beta$, we get

$$
\beta_{x x x}+8 u \beta_{x}+4 u_{x} \beta-T^{\prime-2} \beta_{x}=0,
$$

so that $\beta$ satisfies Lenard's equation [4]. If we use the series expression of $\beta$,

$$
\beta=\sum_{k=0}^{\infty} \beta_{2 k+1} T^{\prime 2 k+1} \quad \text { (see above) }
$$

and the fact that $\beta$ satisfies Lenard's equation, it is easy to see that

$$
\left(\hat{o}^{3}+8 u \partial-T^{\prime-2} \partial+4 u_{x}\right) \sum_{k=0}^{n} \beta_{2 k+1} T^{\prime 2 k+1}=\partial \beta_{2 n+3} T^{\prime 2 n+1} .
$$

If we take $\Sigma_{k=0}^{n} \beta_{2 k+1} T^{\prime 2 k+1}$ for $b_{2}$, we get $u_{t}-\frac{1}{4} \partial \beta_{2 n+3}=0$ after a rescaling of $t$. Here, $n=1$ yields the $\mathrm{KdV}$. The method is inspired by $\operatorname{Lax}[8,10]$.

REMARK. If we use the coadjointed representation of $f, A$ and $B$, it becomes obvious that $\beta$ should satisfy 'Lenard' and $b_{2}$ should be an inhomogeneous version of it. The standard two-dimensional representation almost directly yields the Schrodinger operator.

$N L S$ : The prolongation is described in [12] and the prolongation algebra in [5]. This algebra is $H \times\left(A_{1} \otimes C[T]\right)$, where now $H$ is a three-dimensional commutative Lie algebra. If one proceeds along the lines of the $\mathrm{KdV}$, one finds with the prolongation algebra $\left(A_{1} \otimes \mathbb{C}[T]\right)$ that $\omega=A \mathrm{~d} x+B \mathrm{~d} t$ with

$$
A=\frac{1}{4} T h+\frac{1}{2} \psi y+\frac{1}{2} \bar{\psi} z
$$

and

$$
B=\left(\frac{i}{2} \psi_{x}-\frac{i}{4} T \psi\right) y+\left(-\frac{i}{2} \bar{\psi}_{x}-\frac{i}{4} T \bar{\psi}\right) z+\left(-\frac{i}{8} T^{2}+\frac{i}{4} \psi \bar{\psi}\right) h
$$

For $\alpha, \beta$ and $\gamma$, we find the following analogues of (3) and (4)

$$
\alpha_{x}=-\frac{1}{2} \psi \gamma+\frac{1}{4} T \text { and } \gamma_{x}-\frac{1}{2} T \gamma+\frac{1}{2} \psi \gamma^{2}=\frac{1}{2} \bar{\psi}
$$

with

$$
\alpha_{t}+\gamma\left(\frac{i}{2} \psi_{x}-\frac{i}{4} T \psi\right)=-\frac{i}{8} T^{2}+\frac{i}{4} \psi \bar{\psi}
$$


and

$$
\gamma_{t}+\gamma^{2}\left(\frac{i}{2} \psi_{x}-\frac{i}{4} T \psi\right)+\gamma\left(\frac{i}{4} T^{2}-\frac{i}{2} \psi \bar{\psi}\right)+\frac{i}{2} \bar{\psi}_{x}+\frac{i}{4} T \bar{\psi}=0
$$

Equation ( $\left.3^{\prime}\right)$ can be solved by $\gamma=\sum_{n=1}^{\infty} \gamma_{n} T^{-n}$ [so one works in $\mathbb{C}\left(\left(T^{-1}\right)\right)$ ]. One gets, if $\alpha=-\frac{1}{4} T x+\sum_{n=1}^{*} \alpha_{n} T^{-n}$,

$$
\alpha_{1 x}=\frac{1}{2} \psi \bar{\psi}, \quad \alpha_{2 x}=\psi \bar{\psi}_{x}, \quad \alpha_{3 x}=-\frac{1}{2} \psi^{2} \bar{\psi}^{2}+2 \psi \bar{\psi}_{x x}, \text { etc. }
$$

We must prove now that $\gamma$ satisfies the last equation of $\left(4^{\prime}\right)$. From the second equation of $\left(3^{\prime}\right)$ (which holds true for this $\gamma$ ), it follows that

$$
\left(\frac{\partial}{\partial x}-\frac{1}{2} T+\psi \gamma\right) \gamma_{t}=\gamma^{2}\left(-\frac{i}{2} \psi_{x x}+\frac{i}{4} \bar{\psi} \psi^{2}\right)-\frac{i}{2} \bar{\psi}_{x x}+\frac{i}{4} \psi \bar{\psi}^{2}
$$

where use is made of the NLS and its conjugate.

From this it follows that

$$
\begin{aligned}
& \left(\frac{\partial}{\partial x}-\frac{1}{2} T+\psi \gamma\right)\left(\gamma_{t}+\gamma^{2}\left(\frac{i}{2} \psi_{x}-\frac{i}{4} T \psi\right)+\right. \\
& \left.\quad+\gamma\left(\frac{i}{4} T^{2}-\frac{i}{2} \psi \bar{\psi}\right)+\frac{i}{2} \bar{\psi}_{x}+\frac{i}{4} T \bar{\psi}\right)=0
\end{aligned}
$$

Let $v$ be an analytic function, which is of the form $\Sigma_{n=-\infty}^{m} v_{n} T^{n}$ for $x$ and $t \in \mathbb{R}$, that satisfies

$$
\left(\frac{\partial}{\partial x}-\frac{1}{2} T+\psi \gamma\right) v=0
$$

Then $v_{m}=0$, so $v=0$. It follows that our assertion is proved and that $\alpha_{1 x}, \alpha_{2 x}, \alpha_{3 x}$, etc. are indeed conservation densities. They are also to be found in [13]. Compare the proof with Section 3 of [6].

REMARK. $\gamma \mapsto-\gamma$ gives a trivial Backlund transformation for the NLS: $\psi \mapsto-\psi$. On the other hand, the NLS and its algebra possess a natural $\mathbb{Z}^{2}$-grading (see [5]), the degrees of $x, t, \psi, \bar{\psi}, T$ and $\bar{T}$ being $(-1,0),(-2,0),(1,-1),(1,1),(1,0)$ and $(1,0)$, respectively. The degrees of $\gamma$ and $\bar{\gamma}$ are $(0,1)$ and $(0,-1)$. Indeed:

$$
\gamma \mapsto \frac{1}{\bar{\gamma}} \text { yields } \psi \mapsto \psi-\frac{(T+\bar{T}) \bar{\gamma}}{\gamma \bar{\gamma}-1}
$$

giving another solution of the NLS. 
Burgers: The prolongation 1-form is

$\omega=A \mathrm{~d} x+B \mathrm{~d} t$ with $A=-x_{1}-q x_{2}$ and $B=\left(q_{x}+q^{2}\right) x_{2}+q x_{3}-x_{4}$

where $x_{3}=\left[x_{1}, x_{2}\right]$. The prolongation algebra is presented by the free Lie algebra $L\left(x_{1}, x_{2}, x_{4}\right)$ and the relations

$$
\left[x_{1}, x_{2}\right]+\left[x_{2},\left[x_{1}, x_{2}\right]\right]=0, \quad\left[x_{1},\left[x_{1}, x_{2}\right]\right]-\left[x_{2}, x_{4}\right]=0 \text { and }\left[x_{1}, x_{4}\right]=0 .
$$

See [14].

This presentation problem was solved by Gragert and Martini (both from Twente). The algebra consists of an infinite sequence of letters

or

$$
\begin{aligned}
& \left(x_{n}\right)_{n \geqslant 1} \text { with }\left[x_{i}, x_{j}\right]=0 \text { if } i, j \geqslant 5, \quad\left[x_{3}, x_{n}\right]=0 \text { if } n \geqslant 5, \\
& {\left[x_{1}, x_{3}\right]=x_{5}, \quad\left[x_{1}, x_{n}\right]=x_{n+1} \text { if } n \geqslant 5,\left[x_{n}, x_{2}\right]=x_{n} \text { if } n=3,}
\end{aligned}
$$

$$
n \geqslant 5, \quad\left[x_{3}, x_{4}\right]=x_{6} \text { and }\left[x_{n}, x_{4}\right]=x_{n+2} \text { if } n \geqslant 5
$$

The proof of this is straightforward if one uses the fact that the algebra possesses a $\mathbb{N}$-grading with $\operatorname{deg}\left(x_{1}\right)=1, \operatorname{deg}\left(x_{2}\right)=0$ and $\operatorname{deg}\left(x_{4}\right)=2$. An isomorphic algebra is described in [15]. This algebra is not very tractable but this can be remedied by enlarging it.

Pick two letters $a$ and $b$, give them degree 0 , and let

Set

$$
[a, b]=b, \quad\left[a, x_{2}\right]=b \text { and }\left[b, x_{2}\right]=b .
$$

$$
x_{1}=a \otimes T, \quad x_{4}=-a \otimes T^{2}, \quad x_{3}=b \otimes T \quad \text { and } \quad x_{n}=b \otimes T^{n-3}
$$

for $n \geqslant 5$, where $T$ is an indeterminate. The algebra is now completely described. The intended enlargement is then the three-dimensional algebra $\left(a, b, x_{2}\right)$ over $\mathbb{C}((T))$. If we put $c=x_{2}+a-b$ then $[a, b]=b$ and $[a, c]=[b, c]=0$. So we have two prolongation algebras, commuting with each other, namely $(a, b)$ and $(c)$ both over $\mathbb{C}((T))$.

'Burgers' with $\mathrm{g}=(\mathrm{c})$

Equation (11) can be split up according to the decomposition of the prolongation algebra. For $(c)$ we find

$$
A=-q c \text { and } B=\left(q_{x}+q^{2}\right) c .
$$

This is described in [14].

'Burgers' with $\mathbf{g}=(a, b)$ : We find

$$
A=(-T+q) a-q b \text { and } B=\left(T^{2}-q_{x}-q^{2}\right) a+\left(T q+q_{x}+q^{2}\right) b .
$$

Put $f=\mathrm{e}^{\alpha a} \mathrm{e}^{\beta b}$ and we find

$$
\begin{aligned}
& \alpha_{x}=-T+q \text { and } x_{t}=T^{2}-q_{x}-q^{2}, \\
& \beta_{x}+\beta \alpha_{x}=-q \text { and } \beta_{z}+\beta \alpha_{t}=T_{q}+q_{x}+q^{2} .
\end{aligned}
$$


Put $\alpha^{\prime}=\alpha+T x-T^{2} t$ then we get

$$
\alpha_{x}^{\prime}=q \text { and } \alpha_{i}^{\prime}=-q_{x}-q^{2}
$$

and we have the case with (c) again.

If $Q=\mathrm{e}^{\alpha^{\prime}}$ then $Q_{t}+Q_{x x}=0$ (Cole-Hopf), this follows immediately from (13).

\section{References}

1. Wahlquist, H. D. and Estabrook, F, B., 'Prolongation Structures of Nonlinear Evolution Equations I', J. Math. Phys. 16, 1-7 (1975).

2. Bourbaki, N., Groupes et algèbres de Lie, Chap. II: 'Algèbres de Lie libres', Hermann, Paris, 1972.

3. Ibid., Chap. III: 'Groupes de Lie'.

4. Van Eck, H.N.,'The Explicit Form of the Lie Algebra of Wahlquist and Estabrook. A Presentation Problem', Proc. Kon. Ned. Akad. Wetensch., Series A 86, 149-164 (1983).

5. Van Eck, H. N., Gragert, P. K. H., and Martini, R., 'The Explicit Structure of the Nonlinear Schrödinger Prolongation Algebra', Proc. Kon. Ned. Akad. Wetensch., Series A 86, 165-172 (1983).

6. Miura, R. M., Gardner, C. S., and Kruskal, M. D., 'Korteweg-de Vries Equations and Generalizations, II. Existence of Conservation Laws and Constants of Motion', J. Math. Phys. 9, 1204-1209 (1968).

7. Miura, R. M., 'Korteweg-de Vries Equation and Generalizations, I. A. Remarkable Explicit Nonlinear Transformation', J. Math. Phys. 9, 1202-1204 (1968).

8. Lax, P. D., 'Nonlinear Partial Differential Equations of Evolution', Actes, Congrès Intern. Math.: 1970, Vol. 2, pp. 831-840.

9. Lax, P. D., 'Periodic Solutions of the KdV Equations', in A. C. Newell (ed.), Nonlinear Wave Motion, AMS Proc. 1974, Lect. in Appl. Math., Vol. 15, pp. 85-96.

10. Lax, P. D., 'Almost Periodic Solutions of the KdV Equation', SIAM Rev. 18, 351-375 (1976).

11. Bourbak1, N., Variétés différentielles et analytiques, Hermann, Paris, 1971.

12. Estabrook, F. B. and Wahlquist, H. D., 'Prolongation Structures of Nonlinear Evolution Equations II', J. Math. Phys. 17, 1293-1297 (1976)

13. Kumei, S., 'Group Theoretic Aspects of Conservation Laws of Nonlinear Dispersive Waves: KdV Type Equations and Nonlinear Schrödinger Equations', J. Math. Phys. 18, 256-264 (1977).

14. Kaup, D. J., 'The Estabrook-Wahlquist Method with Examples of Application', Physica D 1D, 391-411 (1980).

15. Krasilshchik, I. S. and Vinogradov, A. M.: 'Nonlocal symmetries and the Theory of Coverings: An Addendum to A. M. Vinogradov's "Local Symmetries and Conservation Laws", Acta Appl. Math. 2, $79-96$ (1984).

16. Gelfand, I. M. and Dikii, L. A., 'Asymptotic Behaviour of the Resolvent of Sturm-Liouville Equations and the Algebra of the Korteweg-de Vries Equations', London Mathematical Society, Lecture Note Series 60, Cambridge University Press, 1981, pp. 13-49. 